\title{
Proton Magnetic Resonance Studies of the Complex of Poly- (2-vinylpyridine) and Bisacetylacetonato Cobalt(II)
}

\author{
Naoki Higuchi, * Toshifumi HiRaOKI, and Kunio Hikichi** \\ Department of Polymer Science, Faculty of Science, \\ Hokkaido University, Sapporo 060, Japan.
}

(Received April 3, 1978)

\begin{abstract}
Interaction between poly(2-vinylpyridine) (P2VP) and bisacetylacetonatocobalt(II) $\left(\mathrm{Co}(\mathrm{acac})_{2}\right)$ in chloroform- $d_{1}\left(\mathrm{CDCl}_{3}\right)$ solution was investigated by means of nuclear magnetic resonance spectroscopy. It was found that NMR signal of H-6 proton of the sidechain pyridine ring of $\mathrm{P} 2 \mathrm{VP}$ shows a remarkable shift to lower fields by the addition of $\mathrm{Co}(\mathrm{acac})_{2}$. The large shift of H-6 proton indicates that the side-chain nitrogen atom is coordinated to the $\mathrm{Co}(\mathrm{acac})_{2}$. The dependence of the paramagnetic shift on the temperature and $\mathrm{Co}(\mathrm{acac})_{2}$ concentration suggests that the chemical exchange between complexed and uncomplexed states of side-chain of P2VP is rapid. The chemical exchange rate is characterized by an activation energy of $13 \mathrm{kcal} / \mathrm{mol}$. The association constant of $1.2 \mathrm{~mol}^{-1}$ and the intrinsic paramagnetic shift of $9.3 \mathrm{ppm}$ were obtained from the concentration dependence of the paramagnetic shift at room temperature for $\mathrm{P} 2 \mathrm{VP}-\mathrm{Co}(\mathrm{acac})_{2}$ complex.

KEY WORDS Poly(2-vinylpyridine)/ Bisacetylacetonato Cobalt(II) / NMR / Paramagnetic Shift /
\end{abstract}

Nuclear magnetic resonance (NMR) is greatly influenced by the presence of paramagnetic species. Because of the dominant magnetic moment of the unpaired electron, significant variations in NMR chemical shifts and relaxation times are observed. A number of NMR studies of paramagnetic complexes have been carried out. ${ }^{1-11}$ It has been found that the investigation of the paramagnetic shift and the relaxation caused by the unpaired electron is very useful in understanding the structure and the behavior of paramagnetic complexes.

Happe and Ward ${ }^{6}$ observed paramagnetic shifts in proton NMR spectra of pyridine-type molecules coordinated with paramagnetic bisacetylacetonato cobalt(II) $\left(\mathrm{Co}(\mathrm{acac})_{2}\right)$. Doddrell and Roberts ${ }^{7}$ also studied the paramagnetic shifts of these complexes. They found that the H-6 proton signals of pyridine and methyl substituted pyridines show the most remarkable shifts when coordinated with $\mathrm{Co}(\mathrm{acac})_{2}$.

\footnotetext{
* Present Address: Institute for Protein Research, Osaka University, Suita 565, Japan.

** To whom all correspondences should be addressed.
}

It is of interest to study the complex formation of a polymer having a pyridine type group as a pendant group and $\mathrm{Co}(\mathrm{acac})_{2}$ by means of NMR. In this report, we wish to present the results of NMR studies on P2VP in a $\mathrm{CDCl}_{3}$ solution containing paramagnetic $\mathrm{Co}(\mathrm{acac})_{2}$.

\section{EXPERIMENTAL}

Commercial 2-vinylpyridine containing a small amount of stabilizer to prevent self polymerization was distilled under reduced pressure $(30 \mathrm{mmHg}$, $82^{\circ} \mathrm{C}$ ). Radical polymerization was initiated by azobisisobutylonitrile in methanol at room temperature. $\mathrm{Co}(\mathrm{acac})_{2}$ was obtained from Wako Chemical Industries, Ltd. It was purified by a sublimation method in an evacuated desiccator containing phosphorus pentoxide at a temperature of $58^{\circ} \mathrm{C}$.

All proton NMR measurements described here were made with a Hitachi R-20B and JEOL PS100 , operating at $60 \mathrm{MHz}$ and $100 \mathrm{MHz}$, respectively.

P2VP and $\mathrm{Co}(\mathrm{acac})_{2}$ were separately dissolved 
in $\mathrm{CDCl}_{3}$. NMR measurements were made soon after both were mixed.

The chemical shift was measured relative to the internal TMS (tetramethylsilane), and the transverse relaxation time $T_{2}$ was calculated using the relation $T_{2}^{-1}=\pi \Delta \nu_{1 / 2}$, where $\Delta \nu_{1 / 2}$ is the half width of the peak.

The total molar concentrations of $\mathrm{P} 2 \mathrm{VP}$ residues and $\mathrm{Co}(\mathrm{acac})_{2}$ are denoted as [P2VP] and [Co(acac $\left.)_{2}\right]$, respectively.

\section{RESULTS AND DISCUSSION}

\section{Paramagnetic Shift}

The low field portions of $100-\mathrm{MHz}$ NMR spectra of P2VP in $\mathrm{CDCl}_{3}$ are shown in Figure 1,<smiles>CCCc1ccccn1</smiles>

(a)

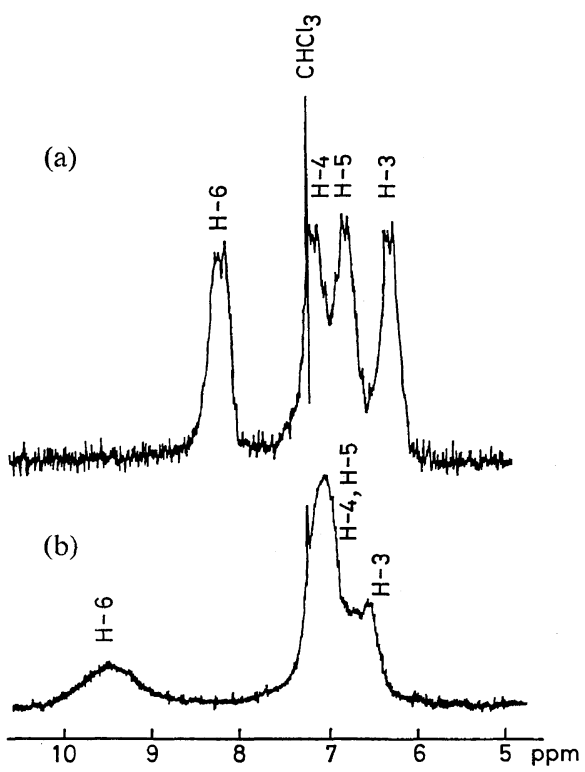

Figure 1. The effect of the addition of $\mathrm{Co}(\mathrm{acac})_{2}$ on the ${ }^{1} \mathrm{H}-\mathrm{NMR}$ spectrum of P2VP at $100 \mathrm{MHz}$ in $\mathrm{CDCl}_{3}$ at room temperature: (A) $[\mathrm{P} 2 \mathrm{VP}]=0.314$ $\mathrm{mol} / l$; (B) $[\mathrm{P} 2 \mathrm{VP}]=0.314 \mathrm{~mol} / l, \quad\left[\mathrm{Co}(\mathrm{acac})_{2}\right]=0.043$ $\mathrm{mol} / l$. in the absence and presence of $\mathrm{Co}(\mathrm{acac})_{2}$ at room temperature. The assignment of the peaks was made by referring to a paper of Weil and Hermann. ${ }^{12}$ The shift and broadening of the resonance lines by the addition of paramagnetic $\mathrm{Co}(\mathrm{acac})_{2}$ are easily observed in Figure 1. Only the peaks of H-3 and H-6 protons of side-chain pyridine ring are considered in this paper, because other peaks are not well resolved.

The peaks of H-6 and H-3 shift down field by increasing the concentration of $\mathrm{Co}(\mathrm{acac})_{2}$ as seen in Figure 2, where the shifts are plotted against

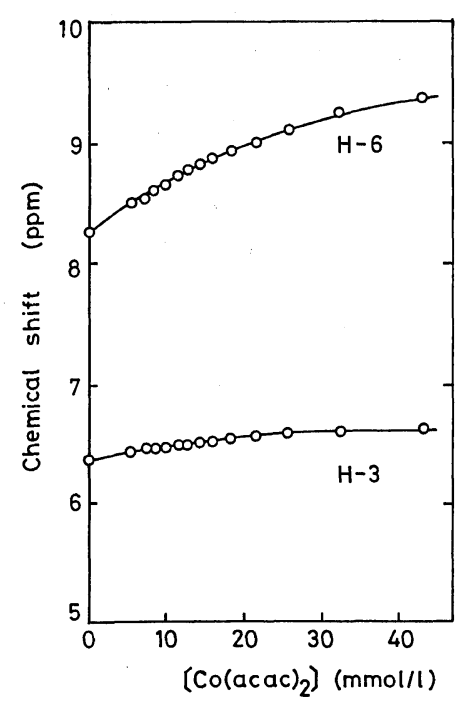

Figure 2. Chemical shifts of H-3 and H-6 protons as a function of the total concentration of $\mathrm{Co}(\mathrm{acac})_{2}$, $\left[\mathrm{Co}(\mathrm{acac})_{2}\right] ;[\mathrm{P} 2 \mathrm{VP}]=0.314 \mathrm{~mol} / \mathrm{l}$, at room temperature. Upper and lower curves show H-6 and H-3 protons, respectively.

the concentration of $\mathrm{Co}(\mathrm{acac})_{2}$ at a constant $\mathrm{P} 2 \mathrm{VP}$ concentration of $0.314 \mathrm{~mol} / l$. The shift induced by $\mathrm{Co}(\mathrm{acac})_{2}$ was found to be considerable for the H-6 than for other protons of the side chain and the main chain. This is in agreement with previous results in which $\mathrm{H}-6$ protons of pyridine and pyridine derivatives show very remarkable paramagnetic shifts. ${ }^{6,7}$

At all $\mathrm{Co}(\mathrm{acac})_{2}$ concentrations studied, the H-6 proton signal is not two separate peaks attributable to complexed and uncomplexed forms, but only a single line, indicating that the rapid chemical exchange occurs between $\mathrm{P} 2 \mathrm{VP}-\mathrm{Co}(\mathrm{acac})_{2}$ complex 
and pure P2VP as in the case of pyridine and pycoline in which the rapid exchange occurs. It is assumed that the complex formation may be written as follows.

$$
\mathrm{P} 2 \mathrm{VP}+\mathrm{Co}(\mathrm{acac})_{2} \rightleftharpoons \mathrm{P} 2 \mathrm{VP}-\mathrm{Co}(\mathrm{acac})_{2}
$$

If the life time of the $\mathrm{P} 2 \mathrm{VP}-\mathrm{Co}(\mathrm{acac})_{2}$ complex, $\tau_{\mathrm{M}}$, is smaller than the reciprocal of the intrinsic paramagnetic shift of the complex, $\Delta \omega_{\mathrm{M}}{ }^{-1}$, and $[\mathrm{P} 2 \mathrm{VP}] \gg\left[\mathrm{Co}(\mathrm{acac})_{2}\right]$, which is satisfied by our experimental conditions, the observed paramagnetic shift $\Delta \omega_{\text {obs }}$ is written as,

$$
\begin{aligned}
\Delta \omega_{\text {obs }} & =P_{\mathrm{M}} \Delta \omega_{\mathrm{M}} \\
& =K \Delta \omega_{\mathrm{M}}\left[\mathrm{Co}(\mathrm{acac})_{2}\right] /(1+K[\mathrm{P} 2 \mathrm{VP}])
\end{aligned}
$$

where $P_{\mathrm{M}}$ is the fraction of $\mathrm{P} 2 \mathrm{VP}-\mathrm{Co}(\mathrm{acac})_{2}$ complex, and $K$ is the association constant of the $\mathrm{P} 2 \mathrm{VP}-\mathrm{Co}(\mathrm{acac})_{2}$. Or

$$
\left[\mathrm{Co}(\mathrm{acac})_{2}\right] / \Delta \omega_{\text {obs }}=1 / K \Delta \omega_{\mathrm{M}}+[\mathrm{P} 2 \mathrm{VP}] / \Delta \omega_{\mathrm{M}}
$$

If we plot $\Delta \omega_{\text {obs }}$ against $\left[\mathrm{Co}(\mathrm{acac})_{2}\right]$ at a constant [P2VP], a straight line is obtained. These results are shown in Figure 3, where plots are made at

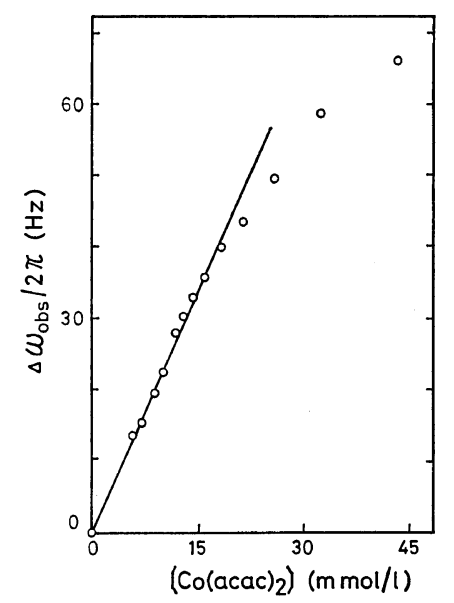

Figure 3. The $\mathrm{Co}(\mathrm{acac})_{2}$ concentration dependence of the H-6 proton paramagnetic shift $\Delta \omega_{\mathrm{obs}}$ at a constant concentration of $[\mathrm{P} 2 \mathrm{VP}]=0.314 \mathrm{~mol} / l$, at room temperature. Measurements were made at $60 \mathrm{MHz}$.

a constant [P2VP] concentration of $0.314 \mathrm{~mol} / \mathrm{l}$ at room temperature. The agreement between the prediction and the experimental results is good at lower concentrations of $\left[\mathrm{Co}(\mathrm{acac})_{2}\right]$, but the deviation from the straight line is apparent at higher concentrations. Figure 4 shows a plot of $\left[\mathrm{Co}(\mathrm{acac})_{2}\right] / \Delta \omega_{\text {obs }}$ vs. [P2VP] at room tempera- ture. We obtained a paramagnetic shift $\Delta \omega_{\mathrm{M}}$ of $9.3 \mathrm{ppm}$ and $K=1.2 \mathrm{~mol}^{-1}$ at room temperature from the slope and the intercept of the straight line of Figure 4. A relatively small value for the association constant seems to indicate that the $\mathrm{P} 2 \mathrm{VP}-\mathrm{Co}(\mathrm{acac})_{2}$ complex is not a very good one.

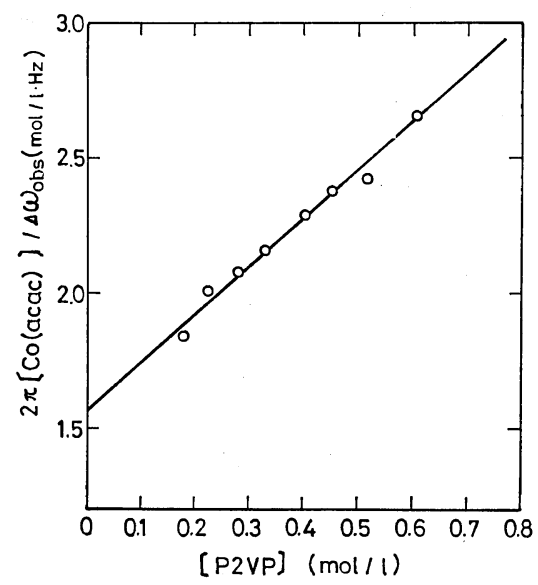

Figure 4. $2 \pi\left[\mathrm{Co}(\mathrm{acac})_{2}\right] / \Delta \omega_{\mathrm{obs}} v s$. $[\mathrm{P} 2 \mathrm{VP}]$ plot, at room temperature.

Figure 5 shows that the paramagnetic shift $\Delta \omega_{\text {obs }}$ at $100 \mathrm{MHz}$ is plotted against the reciprocal temperature at concentrations of $[\mathrm{P} 2 \mathrm{VP}]=0.299$ $\mathrm{mol} / l$ and $\left[\mathrm{Co}(\mathrm{acac})_{2}\right]=0.0209 \mathrm{~mol} / \mathrm{l}$. The fact

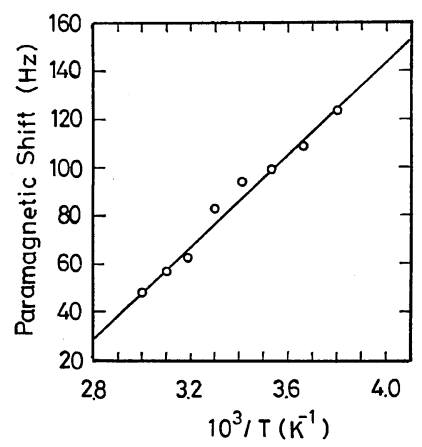

Figure 5. Paramagnetic shift of $\mathrm{H}-6$ proton plotted against the reciprocal temperature at concentration of $[\mathrm{P} 2 \mathrm{VP}]=0.299 \mathrm{~mol} / l$ and $\left[\mathrm{Co}(\mathrm{acac})_{2}\right]=0.0209 \mathrm{~mol} / l$, at $100 \mathrm{MHz}$.

that $\Delta \omega_{\text {obs }}$ decreases by increasing the temperature indicates that the exchange between the complexed and uncomplexed states is fast, i.e., $\tau_{\mathrm{M}} \ll \Delta \omega_{\mathrm{M}}{ }^{-1}$, supporting the validity of the above analysis. 


\section{Transverse Relaxation Rate}

The paramagnetic contribution to the transverse relaxation rate is defined by,

$$
T_{2 \mathrm{p}}^{-1}=T_{2 \mathrm{obs}}^{-1}-T_{20}^{-1}
$$

where $T_{2 \mathrm{obs}}^{-1}$ and $T_{20}^{-1}$ are the transverse relaxation rates in the presence and absence of $\mathrm{Co}(\mathrm{acac})_{2}$, respectively. According to Swift and Connick, ${ }^{3}$ $T_{2 \mathrm{p}}$ is written as,

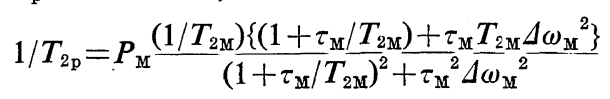

where $T_{2 \mathrm{M}}^{-1}$ is the relaxation rate of the $\mathrm{P} 2 \mathrm{VP}$ $\mathrm{Co}(\mathrm{acac})_{2}$ complex. Due to the very short electronic relaxation time of $\mathrm{Co}(\mathrm{II})\left(\sim 10^{-13} \mathrm{sec}\right) T_{2 \mathrm{M}}^{-1}$ is given by

$$
\begin{aligned}
1 / T_{2 \mathrm{M}}= & \frac{4}{3} \frac{S(S+1) g^{2} \beta^{2} \gamma_{N}{ }^{2}}{r^{6}} \tau_{\mathrm{\theta}} \\
& +\frac{2}{3} \frac{S(S+1) A^{2}}{\hbar^{2}} \tau_{\mathrm{\theta}}
\end{aligned}
$$

where $\tau_{\theta}$ is the electronic relaxation time of Co(II) and $r$ is the distance between the observed proton and the unpaired electron. $S$ is the electron spin quantum number, $g$, the electronic $g$ factor, $\gamma_{N}$, the gyromagnetic ratio of the observed nuclei, $\beta$, Bohr magneton, $\hbar$, Planck's constant divided by $2 \pi$, and $A$, the hyperfine coupling constant. In our case, $\Delta \omega_{\mathrm{M}} T_{2 \mathrm{M}} \gg 1$ is held because the line broadening is not so appreciable that we can observe the paramagnetic shift. Since $\tau_{\mathrm{M}} \Delta \omega_{\mathrm{M}}<1$ as mentioned above, we get

$$
1 / T_{2 \mathrm{p}}=P_{\mathrm{MM}} \tau_{\mathrm{M}} \Delta \omega_{\mathrm{M}}^{2}
$$

This means that the line broadening is essentially caused by the exchange between the two states, and not by the relaxation enhanced by the electron spin.

Combining eq 2 and 7, we obtain

$$
T_{2 \mathrm{p}} \Delta \omega_{\mathrm{obs}}=\left(\tau_{\mathrm{M}} \Delta \omega_{\mathrm{M}}\right)^{-1}
$$

In Figure 6, the logarithms of $T_{2 \mathrm{p}} \Delta \omega_{\mathrm{obs}}$ of the H-6 proton are plotted against the reciprocal temperature at $\left[\mathrm{Co}(\mathrm{acac})_{2}\right]=0.299 \mathrm{~mol} / l$ and $[\mathrm{P} 2 \mathrm{VP}]=$ $0.0209 \mathrm{~mol} / l$. The results indicate that $T_{2 \mathrm{p}} \Delta \omega_{\mathrm{obs}}$ increases with increasing temperature. The temperature dependence of $\Delta \omega_{\mathrm{M}}$ is governed by Curie's law, ${ }^{13}$ while the temperature dependence of $\tau_{\mathrm{M}}$ is probably expressed by the Arrhenius equation. Therefore, it is reasonable that the temperature dependence of $T_{2 \mathrm{p}} \Delta \omega_{\mathrm{obs}}$ is determined primarily by $\tau_{\mathbf{M}}{ }^{-1}$. Thus, we calculated $\tau_{M}$ from eq 8 as-

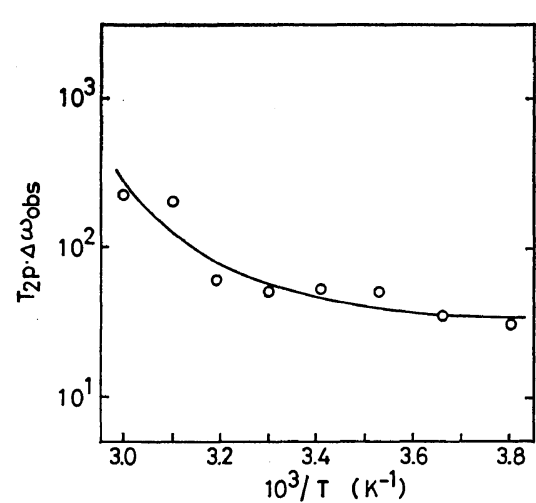

Figure 6. $T_{2 \mathrm{p}} \Delta \omega_{\mathrm{obs}}$ vs. the reciprocal temperature plot of the H-6 peak at $100 \mathrm{MHz} ;[\mathrm{P} 2 \mathrm{VP}]=0.299$ $\mathrm{mol} / l$ and $\left[\mathrm{Co}(\mathrm{acac})_{2}\right]=0.0209 \mathrm{~mol} / l$.

suming that $\Delta \omega_{M}=3510 \mathrm{rad} / \mathrm{s}$ (equivalent to 9.3 $\mathrm{ppm}$ ) is constant at temperatures studied.

Figure 7 shows the temperature dependence of $\tau_{\mathrm{M}}$. The curve apparently deviates from a straight line at low temperatures. This indicates that fast exchange conditions do not hold at lower temperatures. We tried to estimate the activation enthalpy of the exchange at higher temperatures and obtained $13 \mathrm{kcal} / \mathrm{mol}$ which, though not really reliable, does not seem unreasonable.

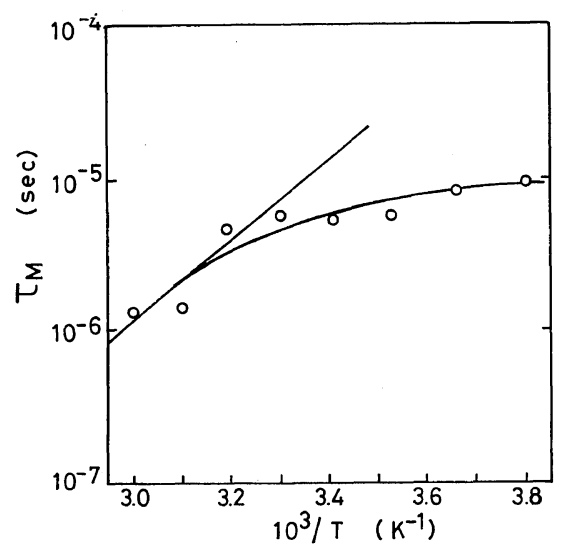

Figure 7. Temperature dependence of the exchange life time $\tau_{\mathrm{M}}$.

In the course of these experiments, the color of the $\mathrm{CDCl}_{3}$ solution of P2VP-Co(acac) $)_{2}$ was a purplish red. But, after one day, it changed to dark green. This was probably due to the change in oxidation number of cobalt from two to three, 


\section{Proton NMR of P2VP-Co(acac $)_{2}$ Complex}

since the $\mathrm{CDCl}_{3}$ solution of $\mathrm{Co}(\mathrm{acac})_{2}$ alone also changes its color after one day following its preparation. The solution of $\mathrm{P} 2 \mathrm{VP}-\mathrm{Co}(\mathrm{acac})_{2}$, following the color change, was found to give a very similar spectra of $\mathrm{P} 2 \mathrm{PV}$ only. Thus, the color change is accompanied by the change from paramagnetic cobalt(II) to diamagnetic cobalt(III).

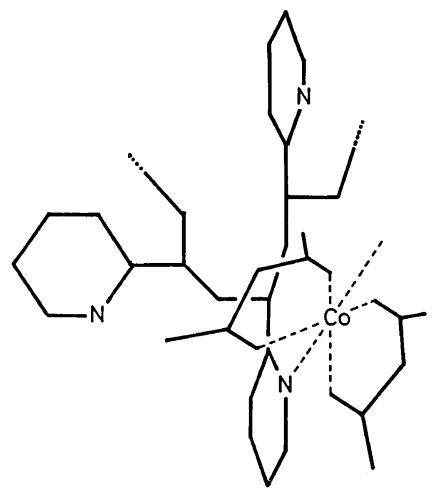

Figure 8. A speculative model of the $\mathrm{P} 2 \mathrm{VP}-\mathrm{Co}(\mathrm{acac})_{2}$ complex.

Finally, in consideration of the model of pyridine- $\mathrm{Co}(\mathrm{acac})_{2},{ }^{6,14}$ we have proposed a speculative model of P2VP-Co(acac $)_{2}$ complex in Figure 8 in which the nitrogen atom of the P2VP sidechain is coordinated to $\mathrm{Co}(\mathrm{acac})_{2}$ apically to the plane of two acetylacetones.

Acknowledgment. The authors wish to thank Dr. A. Tsutsumi, Hokkaido University, for his helpful remarks and advice. This work was supported by the Ministry of Education of Japan.

\section{REFERENCES}

1. W. D. Horrocks, Jr., "NMR of Paramagnetic Molecules," G. N. LaMar, W. D. Horrocks, Jr., and R. H. Holm, Ed., Academic Press, New York, N. Y., 1973.

2) C. C. McDonald and W. D. Phillips, J. Am. Chem. Soc., 85, 3736 (1963).

3. T. J. Swift and R. E. Connick, J. Chem. Phys., 37, 307 (1962).

4. Z. Luz and S. Meiboom, J. Chem. Phys., 40, 1058 (1964).

5. Z. Luz and S. Meiboom, J. Chem. Phys., 40, 1686 (1964).

6. J. A. Happe and R. L. Ward, J. Chem. Phys., 39, 1211 (1963).

7. D. Doddrell and J. D. Roberts, J. Am. Chem. Soc., 92, 6839 (1970).

8. R. G. Shulman, H. Sternlicht, and B. J. Wyluda, J. Chem. Phys., 43, 3116 (1965).

9. H. Sternlicht, R. G. Shulman, and E. W. Anderson, J. Chem. Phys., 43, 3123 (1965).

10. H. Sternlicht, R. G. Shulman, and E. W. Anderson, J. Chem. Phys., 43, 3133 (1965).

11. O. Iwaki, K. Hikichi, M. Kaneko, S. Shimizu, and T. Maruyama, Polym. J., 4, 623 (1973).

12. G. Weill and G. Hermann, J. Polym. Sci., A-2, 5, 1393 (1967).

13. H. M. McConnell and R. E. Robertson, J. Chem. Phys., 29, 1361 (1958).

14. W. D. Horrocks, Jr. and D. D. Hall, Inorg. Chem., 10, 2369 (1971). 